\title{
Factores de riesgo cardiovasculares asociados a pie diabético
}

\author{
Cardiovascular factors associated with diabetic foot
}



${ }^{1}$ Médico cirujano, graduado de la Universidad Autónoma de San Luis Potosí inkvitro@hotmail.com

${ }^{2}$ Dr. Juan Pablo García Ugalde, especialista en Urgencias MédicoQuirúrgicas, Jefe de Urgencias del Hospital General \#2 Delegación IMSS, San Luis Potosí, México

pbo2002@hotmail.com

Revista Médica Sinergia Vol. 4 (3), Marzo 2019

ISSN:2215-4523 / e-ISSN:2215-5279

http://revistamedicasinergia.com

${ }^{1}$ Dr. Juan Manuel López Díaz

Instituto Mexicano del Seguro Social- Hospital General de Zona \#50, México inkvitro@hotmail.com

https://orcid.org/0000-0001-9801-7078

${ }^{2}$ Dr. Juan Pablo García Ugalde Instituto Mexicano del Seguro Social- Hospital General de Zona \#50, México pbo2002@hotmail.com

https://orcid.org/0000-0002-1811-145X

RECIBIDO

$2 / 2 / 2019$

9/2/2019

\section{RESUMEN}

Introducción: el pie diabético una de las complicaciones más frecuentes de la Diabetes mellitus con impacto funcional, económico y elevada morbimortalidad, pudiendo ser evitado hasta en $75 \%$ de casos con modificación sus factores de riesgo; en la literatura mundial la frecuencia de los factores de riesgo cardiovascular en el Pie Diabético son referidos en pocas veces de manera individual, por lo que su correcta identificación daría una base más sistemática para su abordaje.

Objetivo: identificar los Factores cardiovasculares en pacientes con Diabetes mellitus que están asociados a pie diabético.

Material y métodos: el estudio es observacional, analítico con diseño trasversal, en pacientes adultos en área de urgencias con diagnóstico de Diabetes mellitus. Se estudiaron las variables: presencia o ausencia de pie diabético, edad, género, hipertensión, sobrepeso, tabaquismo, Dislipidemia. Mediante R Studio 1.0.153, se determinó normalidad, se realizaron correlaciones y comparaciones entre variables.

Análisis estadístico: se obtuvieron 201 pacientes, media de edad: $59.4 \pm 15.3$ años, $48.76 \%$ de género masculino y $51.24 \%$ femenino; prevalencia de pie Diabético de $31.34 \%$ (63 pacientes). Se analizó cada variable, siendo no estadísticamente significativas: edad $(p=0.2172)$, Sexo $(p=0.8796)$, sobrepeso $u$ Obesidad ( $p=0.1423)$, Tabaquismo $(p=0.4993)$ y dislipidemia $(p=0.8699)$. Existió una asociación estadísticamente significativa para hipertensión mediante Prueba Exacta de Fisher (p: 0.001156), con intervalo de confianza de 95\% (1.556-9.603) y Odds Ratio de 3.6456.

Conclusiones: de las variables señaladas como factores de riesgo cardiovascular solo la hipertensión arterial sistémica presento correlación estadísticamente significativa con el pie diabético.

PALABRAS CLAVE: hipertensión; factores de riesgo; pie diabético.

\section{ABSTRACT}

Introduction: diabetic foot is one of the most frequent complications of Diabetes mellitus with functional impact and high morbidity and mortality, being able to be 
avoided in up to $75 \%$ of cases with modification of risk factors; In the world literature, the frequency of cardiovascular risk factors in the Diabetic Foot are referred in a few times individually, so that their correct identification would give a more systematic basis for their approach.

Objective: to identify the cardiovascular factors in patients with diabetes mellitus that are associated with diabetic foot.

Material and methods: the study is observational, analytical with transversal design, in adult patients in emergency area diagnosed with Diabetes mellitus. The variables were studied: presence or absence of diabetic foot, age, gender, hypertension, overweight, smoking, dyslipidemia. Using R Studio 1.0.153, normality was determined, correlations and comparisons between variables were made.

Statistical analysis: 201 patients were obtained, mean age: $59.4 \pm 15.3$ years, $48.76 \%$ male and $51.24 \%$ female; Diabetic foot prevalence of $31.34 \%$ (63 patients). Each variable was analyzed, being not statistically significant: age ( $p=$ $0.2172)$, Sex $(p=0.8796)$, overweight or Obesity $(p=0.1423)$, Smoking ( $p=$ 0.4993 ) and dyslipidemia ( $p=0.8699$ ). There was a statistically significant association for hypertension by Fisher's Exact Test ( $p$ : 0.001156), with 95\% confidence interval (1556-9.603) and Odds Ratio of 3.6456.

Conclusions: of the variables indicated as cardiovascular risk factors, only systemic arterial hypertension showed a statistically significant correlation with diabetic foot.

KEYWORDS: hypertension; risk factors; diabetic foot.

\section{INTRODUCCIÓN}

La diabetes mellitus (DM) corresponde a una alteración metabólica caracterizada por la presencia de hiperglucemia secundaria a la secreción inadecuada de insulina, alteraciones en su acción u ambos; es clasificada en 4 categorías generales:

1. DM tipo 1 (secundario a la destrucción de células- $\beta$ pancreáticas conllevando una deficiencia absoluta de insulina).

2. DM Tipo 2 (debido a una pérdida progresiva de la secreción de insulina aunado a resistencia insulínica).

3. DM gestacional (diagnosticada en el segundo o tercer trimestre de embarazo).

4. Relacionada a otras causas:
síndromes diabéticos monogénicos (
diabetes neonatal, tipo MODY), patologías
de páncreas exocrino(fibrosis quística),
relacionada a drogas(uso de
glucocorticoides, tratamiento para
VIH/SIDA o después de trasplantes de
órganos (1).
El pie diabético (PD) es definido como la
infección, ulceración y/o destrucción de

tejidos profundos asociado a alteraciones neurológicas y varios grados de enfermedad vascular periférica de miembros inferiores de pacientes con DM. Esta enfermedad relacionada a miembros pélvicos está dentro de las más serias y costosas complicaciones dentro del espectro de DM, que al estar presentes pueden resultar en la formación de ulceras y la subsecuente amputación de la extremidad inferior afectada (2).

\section{EPIDEMIOLOGÍA}

La DM es una patología devastadora de distribución mundial, en 1985 se estimaban 30 millones de personas diagnosticadas, en 2000 se suponían 150 millones y para el 2012 la International Diabetes Federation. estima más de 371 millones de personas afectadas, se proyecta que para el 2030 aumente a 552 millones que igualaría a 3 nuevos casos por segundo (3).

El PD es uno de los problemas más devastadores de la DM, aumentando hasta 15 veces el riesgo de amputaciones comparado con población en general. 
Aproximadamente $20 \%$ de los pacientes diabéticos presentaran un cuadro de PD en algún momento de su vida y de éstos el $20 \%$ terminara en amputación (4). Por lo que se estima que el $75 \%$ de todas las amputaciones no traumáticas están relacionadas a DM y de éstos pacientes amputados de un miembro pélvico el $50 \%$ a los 5 años perderán la extremidad inferior contralateral por la misma causa, ocasionando incapacidad temporal o definitiva con tratamiento prolongado y de alto costo (5).

\section{FACTORES DE RIESGO PARA PIE DIABÉTICO}

No todos los pacientes con diabetes tienen probabilidad para desarrollar ulceraciones (las cuales son la mayor complicación de la $\mathrm{DM}$, que en la mayoría de los casos preceden a infecciones y amputaciones con alta morbilidad, mortalidad y costos), por lo que en general pacientes sin factores de riesgo parecieran no estar relacionados con la aparición de ulceras. Se define como pacientes en riesgo a: pacientes con DM que no tienen una ulcera activa en pie, pero cursan con neuropatía periférica, con o sin presencia de deformidad o enfermedad arterial periférica (EAP)o historia de ulcera u amputación de una parte del pie o pierna (6).

Los factores para desarrollar úlceras o amputación en pacientes con DM son (Ver CUADRO 1. 1):

CUADRO 1. Factores de riesgo para ulceración o amputación de pie diabético

- Diabetes de más de 10 años de evolución

- Sexo masculino

- Neuropatía diabética

- Insuficiencia arterial

- Deformidad de pie con evidencias de sitios de presión, o callosidad plantar severa

- Patología de uñas

- Historia previa de úlcera o amputación

Fuente: Tratamiento de la Diabetes Mellitus tipo 2 en el primer nivel de el primer nivel de Atención. Instituto Mexicano del Seguro Social. 2014 (7)

\section{FACTORES DE RIESGO CARDIOVASCULAR EN PIE DIABÉTICO}

Los pacientes con PD tienen una más alta prevalencia de factores de riesgo cardiovascular (FRC) como hipercolesterolemia, hipertrigliceridemia y microalbuminuria con una elevada prevalencia de enfermedad cardiovascular (enfermedad coronaria [EC], eventos cerebrales $[E V C]$ isquémicos, ataque cerebral isquémico transitorio [AIT] y retinopatía diabética $[R D])$. Agregado a esto, individuos con PD ulcerado han mostrado después de 5 años de seguimiento una alta incidencia de nuevos eventos vasculares incluyendo: EC, AIT, EVC isquémicos y RD. (8). Esto es relacionado con el hecho que la DM tiene un complejo nexo de variables con alteraciones inflamatorias metabólicas y consecuente efecto sobre el sistema cardiovascular que explicaría los reportes de alta morbi-mortalidad previo en pacientes diabéticos con amputaciones. La hipertensión arterial (HTA) y la DM representan 2 FRC independientes, ambos aceleran el desarrollo de RD, nefropatía y enfermedad vascular periférica (EVP). En todos los casos la HTA empeora el pronóstico aumentando el riesgo de complicaciones tanto macrovascular $y$ microvasculares relacionadas ambas al PD. En adición, se muestran altas prevalencias de dislipidemia (DL) en pacientes con PD ulcerado que en aquellos que no cursan con PD, esto en el contexto de que la DL es un factor que incrementa el riesgo de enfermedades macrovasculares en población diabética (9).

En los pacientes con DM2 se tiene conocido que cursan con un alto riesgo de mortalidad relacionada a enfermedad cardiovascular aunado a esto las ulceras del PD indican una coexistente EAP y arterioesclerosis difusa que durante su tratamiento sufren de estrés fisiológico (relacionado con dolor en heridas, infecciones, inflamación severa secundario 
a aumento de citosinas inflamatorias) y físico(ansiedad relacionada a amputaciones) que puede provocar isquemia coronaria severa y deterioro de la función cardiaca aumentando el riesgo cardiovascular (10).

Desglosando los FRC implicados en el PD, están relacionados con los siguientes mecanismos:

- La hipertrigliceridemia es un factor conocido de lesión microvascular, los niveles elevados han mostrado ser un factor de riesgo independiente para amputación de miembros pélvicos en pacientes diabéticos, relacionado con estrés oxidativo, disfunción mitocondrial y elevación de los marcadores inflamatorios con afección directa a nivel vascular.

- La obesidad ejerce sus efectos a nivel metabólico e inflamatorio llevando a alteraciones microvasculares y lesión a nivel de nervios periféricos, secundario a la elevación de citosinas proinflamatorias (interleucinas, factor de necrosis tumoral, lipoproteínas de baja densidad oxidadas, factor de necrosis tumoral) y disminución de citosinas antiinflamatorias (adiponectina, grelina, e interleucina 10) (11).

\section{Los efectos cardiovasculares} relacionados al tabaquismo (definido como: consumo de 1-4gr de tabaco al día 0 en consumidores ocasionales al menos 100 cigarros totales en a lo largo de la vida) están relacionados aun en dosis menores, refiriendo que "no hay un nivel seguro de exposición al tabaco" (12), con injuria directa a nivel endotelial, a través de un incremento de estrés oxidativo, desacoplamiento del óxido nítrico sintetasa, con una posterior perdida de respuesta en la función vascular $y$ de las propiedades antiagregantes endoteliales, activación de plaquetaria y leucocitaria aumentando la disfunción celular endotelial (13).

\section{PATOGÉNESIS DEL PIE DIABÉTICO}

La patogénesis del PD resulta de la acción de múltiples factores siendo una de las principales causas a remarcar: la neuropatía periférica e isquemia resultante de la EVP. La neuropatía periférica está relacionada con más del $60 \%$ de pacientes con PD causada por alteraciones metabólicas inducidas por el estado de hiperglucemia, llevando al incremento de la acción enzimas como aldosa reductasa y sorbitol deshidrogenasa (aumenta la conversión de glucosa a fructosa y sorbitol respectivamente); la acumulación de productos derivados de glucosa resulta en un decremento de la producción inositol en las células nerviosas, el cual es requerido para la conducción neuronal normal (neuropatía). Adicionalmente, la conversión de glucosa resulta de una depleción de las reservas de dinucleótido de nicotinamida y adenina fosfato (ADP) el cual es necesario para para la desintoxicación de las especias reactivas de oxígeno y la síntesis de óxido nítrico (vasodilatador) incrementando tanto el estrés oxidativo en la célula nerviosa y la vasoconstricción llevando a isquemia, promoviendo la lesión y muerte de la célula nerviosa. La hiperglucemia es relacionada con el aumento de con un factor de agregación plaquetaria y ponte vasoconstrictor: tromboxano A2 que aumenta el riesgo de hipercoagulabilidad plasmática llevando a alteraciones en la matriz extracelular vascular e incrementando el riesgo de colapso de la luz arterial. Por tanto, la EAP contribuye en el desarrollo de ulceras en un $50 \%$, con afección más en arterias tibiales y del peroné, debido a la disfunción endotelial y alteraciones de las células musculares lisas debidas a la persistencia del estado hiperglucémico (12).

Las alteraciones anatómicas y estructurales son divididas en 3 tipos:

- Alteraciones sensitivas: es el principal y más común predictor de ulceración (hasta $78 \%$ de los casos) 
daño en la sensibilidad implica la falta de dolor a la deambulación con retardo de la percepción de úlceras por la persona afectada con disfunción de cicatrización en heridas aumentando el área afectada traumatizada.

- Alteraciones motoras: presentación de anormalidades en la anatomía dinámica del pie, causando debilidad y deterioro de la musculatura intrínseca modificando su presión plantar al deambular, llevando a reducción de movimiento y cambios en articulaciones, musculatura y tejidos blandos propios del pie; como ejemplos: dedos en garra, pie cavo, deformación equina, deformaciones ungueales, y como mayor colapso mecánico la neurosteoartropatía de Charcot.

- Alteraciones autonómicas: resulta en una disminución de la inervación autónoma de las glándulas sudoríparas de la piel causando resequedad, disminución de elasticidad principalmente desde tercio medio de la pierna, aumentando la frecuencia de fisuras, grietas cutáneas y formación de callosidades y así aumentando el riesgo de infecciones de heridas (13).

La infección del PD junto con la isquemia, neuropatía y el edema constituyen una tétrada de factores agravantes que incrementan la morbilidad y muerte, el avance de ésta última es el resultado de diversos factores asociados con la característica de la herida, virulencia de la bacteria y el estado del hospedero. El principal factor predisponente de infección es la ulceración frecuentemente asociada con la neuropatía. El desencadenante es externo el cual puede ser físico, químico o mecánico (14).

El camino hacia la ulceración sigue posterior al trauma de los pacientes con insensibilidad por neuropatía diabética del pie, la cual además pudiera estar mal perfundida debida a la EAP siendo más difícil su recuperación.

La ulceración e infección incrementaran la demanda de oxígeno, aunado a que los procesos de reparación están desacoplados por varios procesos inherentes a la diabetes incluyendo a la actividad inmunológica humoral y respuesta inflamatoria anormal. Las alteraciones microvasculares incluyen el incremento de shunts arteriovenosos y una deficiente vasoreactividad, además de una formación deficiente de vasos colaterales, en los cuales llevaran a hipoperfusión capilar incrementando la incapacidad para sanar. Consecuentemente las oclusiones arteriales pueden resultar en un agravamiento extra de la perfusión. Al final la distribución de la EVP en pacientes con diabetes es distal y difusa con alta prevalencia de oclusiones creando un círculo junto con la neuropatía (15).

\section{MANIFESTACIONES CLÍNICAS DEL PIE DIABÉTICO}

La presentación del PD comprende una variedad de síntomas y signos desde locales a sistémicos. Los signos locales de infección pueden incluir dolor/insensibilidad, eritema, edema, salida de material purulento, fetidez. Por parte de los signos sistémicos de infección incluyen anorexia, nausea, vómito, fiebre, sudoración nocturna, alteración del estado mental y alteración de control glucémico. Heridas con purulencia y 2 o más signos locales de inflamación como aumento de temperatura local, eritema, linfangitis, linfadenopatía, edema, dolor o alteraciones de la sensibilidad pueden ser clasificadas como infectadas.

La insuficiencia vascular de los miembros pélvicos es identificada por uno o más de los siguientes signos 0 síntomas: claudicación, dolor en reposo, ausencia de pulsos, rubor, atrofia tegumentaria, palidez a la elevación del miembro, por lo que el PD puede ser dividido en 2 grupos mayores teniendo en cuenta la descripción anterior en: $P D$ isquémico o PD neuropático no isquémico (16). 


\section{CLASIFICACIÓN DEL PIE DIABÉTICO}

La clasificación del PD es necesaria para varios propósitos, una de los más importantes es la descripción de las lesiones para su tratamiento y su estadificación para el contexto clínico y de investigación. La Clasificación de WagnerMeggitt's (TABLA 1.1) fue desarrollada en los 1970 s ha sido la más ampliamente aceptada de manera universal, usada para estadificar lesiones de pie diabético, consta de 6 grados, sin embargo, no toma en cuenta adecuadamente las infecciones, es limitado a identificar alteraciones vasculares.

\begin{tabular}{|l|l|}
\hline \multicolumn{2}{|l|}{ TABLA 1.1 Clasificación de Wagner } \\
\hline Grado 0 & Unicamente dolor \\
\hline Grado 1 & Ulcera superficial \\
\hline Grado 2 & Ulcera profunda \\
\hline Grado 3 & Ulcera que involucra hueso \\
\hline Grado 4 & Gangrena parcial de pié \\
\hline Grado 5 & Gangrena total de pié \\
\hline
\end{tabular}

Fuente: Torres FM. Diagnóstico, clasificación y tratamiento de las infecciones en pie diabético. Cirugia general (17)

TABLA 1. 2. Clasificación de la Universidad de Texas.

\begin{tabular}{|c|c|c|c|c|}
\hline & Grado 0 & Grado 1 & Grado 2 & Grado 3 \\
\hline $\begin{array}{l}\text { Estadio } \\
\text { A }\end{array}$ & $\begin{array}{l}\text { Lesión pre } \\
\text { ó post - } \\
\text { ulcerativa } \\
\text { completam } \\
\text { ente } \\
\text { epitelizada }\end{array}$ & $\begin{array}{l}\text { Herida } \\
\text { superfici } \\
\text { al que no } \\
\text { afecta } \\
\text { tendón, } \\
\text { cápsula } \\
\text { o hueso }\end{array}$ & $\begin{array}{l}\text { Herida } \\
\text { penetra a } \\
\text { tendón o } \\
\text { cápsula }\end{array}$ & $\begin{array}{l}\text { Herida } \\
\text { penetra a } \\
\text { hueso u } \\
\text { articulació }\end{array}$ \\
\hline $\begin{array}{l}\text { Estadio } \\
\text { B }\end{array}$ & Infección & Infección & Infección & Infección \\
\hline $\begin{array}{l}\text { Estadio } \\
\text { C }\end{array}$ & Isquemia & Isquemia & Isquemia & Isquemia \\
\hline $\begin{array}{l}\text { Estadio } \\
\text { D }\end{array}$ & $\begin{array}{l}\text { Infección e } \\
\text { isquemia }\end{array}$ & $\begin{array}{l}\text { Infección } \\
\text { e } \\
\text { isquemia }\end{array}$ & $\begin{array}{l}\text { Infección } \\
\text { e } \\
\text { isquemia }\end{array}$ & $\begin{array}{l}\text { Infección } \\
\text { e } \\
\text { isquemia }\end{array}$ \\
\hline
\end{tabular}

La Clasificación de la Universidad de Texas (TABLA 1. 2) utiliza 4 grados modificados por la presencia de infección, isquemia u ambos, con más visión hacia al pronóstico en el cual a estadios más elevados son menos capaces de mejorar sin revascularización o amputación (17).

\section{TRATAMIENTO DEL PIE DIABÉTICO}

Patología no ulcerativa: en un paciente de alto riesgo, las alteraciones de uñas, piel y callosidades deben de ser tratados periódicamente, de manera preferible por un especialista en el cuidado del pie. De ser posible las deformidades podálicas debieran ser resueltas de manera no quirúrgica. Un calzado inadecuado es una de las mayores causas de ulceración, el indicado debe de ser usado continuamente adaptado para tratar las deformidades y alteraciones biomecánicas.

Patología ulcerativa: pacientes con la presencia de ulcera más profunda al tejido subcutáneo necesita tratamiento intensivo y dependiendo de los recursos hospitalarios considerar su ingreso hospitalario. Para el manejo se consideran los siguientes principios (18):

a) Manejo de puntos de presión / protección de úlceras: liberación de presión mecánica, uso de calzado temporal adaptado, uso de plantillas individuales, limitación de cargas pesadas y sedestación prolongada.

b) Restauración de perfusión: considerar revascularización en pacientes con signos o síntomas de isquemia, énfasis en la prevención de factores de riesgo cardiovascular.

\section{c) Tratamiento de infección}

Úlcera superficial: curación y debridación de tejido necrótico, inicio de antibioticoterapia empírica contra S. Aureus y Estreptococo.

Úlcera profunda: evaluación urgente para drenaje quirúrgico con remoción de tejido necrótico incluyendo hueso infectado $y$ drenaje de abscesos, considerar revascularización arterial, uso de 
antibioticoterapia empírica parenteral de amplio espectro con objetivos hace bacterias gram positivas y negativas incluyendo anaerobios.

d) Control metabólico: control glucémico óptimo con objetivo menor de $140 \mathrm{mg} / \mathrm{dl}$, tratamiento de edema y desnutrición.

e) Cuidado local: inspección frecuente, debridación frecuente con uso de bisturí en caso necesario, consideración de sistemas de presión negativa en heridas postquirúrgicas.

\section{PREVENCIÓN DEL PIE DIABÉTICO}

- Cuidado integrado: se sugiere manejo que incluya personal podólogo o en adición con equipo multidisciplinario(endocrinólogo,

personal de enfermería) para la detección y tratamiento de PD, incluyendo siempre atención a eliminación de callosidades, manejo en cuidado de uñas, educación del paciente y prescripción de calzado terapéutico con explicación de su uso. La educación del paciente puede ser por diferentes métodos (individuales 0 sesiones de grupo), diferentes intervalos (única o semanales) o por diferentes educadores (médicos, enfermería, podólogo), con disminución de úlceras en pacientes que sí se adhieren a realización de cambios en su estilo de vida. El uso de calzado terapéutico puede ser efectivo en la prevención de ulceras plantares en comparación con sólo cuidados básicos del pie. La frecuencia de atención con profesional varía de 1 a 6 meses.

- Autocuidado: intervenciones por el propio paciente desde su educación, inspección de pies frecuente para rápido reconocimiento de patologías agregadas y modificaciones de estilo de vida. Uno de los puntos con más validez es el reconocimiento de alteraciones de temperatura podálica con el subsecuente uso de acciones preventivas para evitar el riesgo de formación de primeras ulceras 0 recurrentes (19).

\section{JUSTIFICACIÓN}

Dentro de las patologías crónicas más frecuentes en pacientes con DM están las enfermedades cardiovasculares (comprendiendo la HTA, enfermedades coronarias e insuficiencia cardiaca) (20). El PD es una de las complicaciones que se presenta en pacientes con DM (en promedio 5 años de evolución desde el diagnóstico), siendo la causa más frecuente de amputación no traumática en pacientes mayores de 50 años, aumentando los costos en salud tanto por la pérdida de la capacidad funcional en personas físicamente activas y secundario al tratamiento médico-quirúrgico intrahospitalario (21). Por otra parte, la DM como patología independiente aumenta por si sola el riesgo de amputaciones y muerte en población general (22). Aunado a esto, la ulceración del PD está asociado a un alta morbi-mortalidad y representando la causa principal de hospitalizaciones en pacientes diabéticos. El riesgo de ulceración de por vida es del $15-25 \%$ con incidencia anual de $2 \%$ y estimación de riesgo de amputación 23 veces mayor comparado con una persona sin DM (23).

La EVP (factor de riesgo independiente para amputaciones) está presente en un $50 \%$ de los casos de PD ulcerado, siendo uno de los pilares de la patología del PD, estando relacionada directamente con los FRC (establecidos por patología vascular incipiente) (23).

Se ha reportado que la morbi-mortalidad de la enfermedad cardiovaular es de 2 a 4 veces mayor en pacientes con DM en comparación a pacientes no diabéticos, lo cual es también relacionado con la presencia y alta prevalencia de los FRC en pacientes con PD, siendo los principales: hipercolesterolemia, hipertrigliceridemia e HTA (factor de riesgo independiente para EVP) (24) y en últimas estudios: la asociación de tabaquismo y como FRC con 
aumento de probabilidad de amputaciones relacionado a la EAP. (25).

En recientes bibliografías se documenta que el porcentaje de prevención de complicaciones de PD (úlceras) puede llegar a ser hasta un $75 \%$ mediante manejo multidisciplinario (26). Por lo que a nivel mundial las estrategias de prevención, detección y tratamiento de los factores de riesgo para PD han demostrado abatir los índices de amputación y mortalidad (consecuencia de la poca difusión entre médicos de primer nivel y de mala divulgación hacia los pacientes) evitando los efectos deletéreos en la población con DM mejorando las condiciones de bienestar (21).

Por lo que este estudio ampliaría la información acerca de la cantidad de pacientes ingresan a servicio de urgencias con el diagnostico de PD (hasta ahora solo englobado en estadísticas internas del hospital como complicaciones vasculares periféricas sin hacer hincapié a PD únicamente) independientemente del motivo de ingreso al servicio, con una estimación más precisa del elevado costo por su atención; además con el objetivo principal de identificar la prevalencia individual de cada FRC relacionados con PD en vista al manejo dirigido para su prevención desde primer nivel de atención médica.

EI PD por trascendencia poblacional es un campo que requiere hincapié en su investigación para su adecuado manejo. Múltiples organizaciones han sido fundadas por esta razón, a pesar de que el progreso en el entendimiento de la etiología, fisiopatología y tratamiento aún se necesita los puntos clave para prevención y óptimo manejo. En el PD los FRC son englobados en la EAP, incluyendo la HTA y tabaquismo los cuales están establecidos para prevenir la progresión de la misma EVP (27). El manejo conservador del PD ha reducido dramáticamente el riesgo de amputación con el uso de medidas primarias de prevención. Las complicaciones del PD son una problemática altamente prevenible, en donde la educación del paciente es esencial; donde el reconocimiento global de la patología como los factores de riesgo toman parte para su comprensión, manejo e impacto (28).

En la actualidad solo existe una base bibliográfica publicada por Pinto et al. En el año 2008 exponiendo el perfil de FRC en pacientes con PD versus pacientes con DM sin PD, con una población total de 225 pacientes realizada en Europa (Italia) (8) con el resto de las publicaciones existentes solo hace referencia a FRC en general sin hablar de la prevalencia individualmente.

\section{OBJETIVOS}

Objetivo general: determinar la asociación de los FRC en pacientes con DM asociados a pie diabético.

Objetivos específicos: establecer la frecuencia de presencia de PD en pacientes con DM que acudan urgencias.

- Determinar la asociación de hipertensión con el pie diabético.

- Determinar la asociación de sobrepeso con pie diabético.

- Determinar la asociación de la hipercolesterolemia con el pie diabético.

- Determinar la asociación del tabaquismo con el pie diabético.

- Determinar la asociación de la hipertrigliceridemia con el pie diabético.

\section{MATERIAL Y MÉTODOS}

1. Tipo de Estudio: observacional, analítico, trasversal

2. Diseño: trasversal

3. Universo de estudio: Hospital General de Zona \#50 (HGZ \#50 )IMSS San Luis Potosí

4. Unidad de investigación: pacientes hospitalizados en el servicio de urgencias del HGZ \#50 con diagnóstico de DM

5. Límite de Tiempo: febrero 2018 febrero 2019

6. Espacio: HGZ \#50 San Luis Potosí, SLP, área de urgencias 
7. Técnica de muestreo: no probabilístico, determinado por criterios de inclusión.

8. Tamaño de la Muestra: para el cálculo del tamaño de la muestra se empleó un modelo de regresión logística: pie diabético (presente/ausente) HTA + sobrepeso + hipercolesterolemia + tabaquismo + hipertrigliceridemia.

El modelo suma 5 grados de libertad tomando, la frecuencia de pie diabético reportada en la literatura es de un 20\% (29), calculándose así un mínimo de 250 y un recomendado de 500 pacientes.

9. Análisis estadístico: se realizó tamaño de muestra mediante modelo de regresión logística que de acuerdo con frecuencia de PD reportada siendo del $20 \%$ con 5 grados de libertad para un cálculo de 250 pacientes como mínimo; posteriormente para la estadística descriptiva, las variables continuas se expresan en promedio +/desviación estándar o como mediana y rangos cuando no cumplan los requisitos de normalidad, las categóricas como proporciones. Las variables categóricas se analizarán mediante prueba exacta de Fisher y las continuas con t de Student o $\mathrm{U}$ de Mann Whitney el nivel de significancia será $p=<0.05$. Se realizará un análisis de regresión logística con el programa $\mathrm{R}$ con el paquete Rcommander

\section{CRITERIOS DE INCLUSIÓN Y EXCLUSIÓN}

\section{Criterios de inclusión}

- Sexo indistinto

- Pacientes con diagnóstico de diabetes mellitus

- Pacientes de 18 años o mayor

- Pacientes que acudan a servicio de urgencias

\section{Criterios de exclusión}

- Pacientes obstétricas o puérperas
- Pacientes con enfermedades reumatológicas

- Pacientes que no aceptan firmar consentimiento informado

- Pacientes con amputaciones de miembros pélvicos o torácicos previas

- Reingresos a servicio de urgencias

\section{Criterios de eliminación}

- Pacientes con datos a recabar incompletos

- Pacientes que no acepten participar o abandono en cualquier momento del estudio

\section{VARIABLES}

\section{Variable independiente}

- Hipertensión

- Tabaquismo

- Sobrepeso

- Hipertrigliceridemia

- Hipercolesterolemia

\section{Variable dependiente}

- Pacientes diabéticos con pie diabético

\section{TÉCNICA DE REALIZACIÓN}

\section{Procedimiento}

1. Elaboración del protocolo, y se solicitará la carta de no inconveniente para realizar este estudio en el HGZ \#50 firmada por la directora y el Coordinador clínico de educación en salud, dando su visto bueno.

2. Se enviará el protocolo al comité de ética e investigación en salud para su evaluación y aceptación.

3. Para el estudio se requiere en un mínimo de 250 pacientes a un recomendado de 500 pacientes que cumplieran con los criterios de inclusión a los cuales después de firmar consentimiento informado se continuara con realización de cuestionario de datos personales y patología diabética. 
4. Se elegirán a todos los pacientes que ingresen a servicio de urgencias que cuenten con el diagnostico de diabetes mellitus (definición en cuadro de variables) que cumplan los criterios de inclusión, sin presencia de criterios de exclusión, que previamente informados acepten ingresar a protocolo de estudio con posterior firma consentimiento informado.

5. Se procederá a realizar mediciones antropométricas tanto medición de talla, peso como se describe a continuación. Medición de peso: se comprueba el adecuado funcionamiento de la báscula de plataforma dentro del servicio de urgencias, se verifica que las vigas de kilogramos y fracciones se encuentren en cero y la báscula se encuentre bien balanceada. Se procede a colocar al paciente (previo retiro de objetos innecesarios que puedan modificar su peso real, quedando con la mínima cantidad de ropa ligera posible) en el centro de la plataforma, de frente al medidor, erguido con hombros abajo, talones juntos y puntas separadas, sin movilizar al paciente para evitar oscilaciones de la lectura de peso, se continua con el registro de peso al deslizar la viga de kilogramos al peso estimado y ajustar con mismo movimiento deslizante la viga de fracciones para el gramaje hasta que la báscula de balance marque cero, haciendo la lectura del peso del paciente y registrándola en kilogramos en hoja de datos individual. Medición de altura: se realizara con estadímetro de báscula de plataforma ya utilizada en la medición de toma de peso, con el paciente de frente al medidor, erguido con hombros abajo, talones juntos y puntas separadas (previo retiro de su calzado que modifique altura medida) con cabeza firme y recta; se procede a medición de altura desliando el estadímetro de arriba hacia abajo hasta topar con la cabeza del paciente presionando suavemente, realizando la lectura con los ojos en el mismo plano horizontal que marca la ventana del estadímetro y registrándola con exactitud en metros en su hoja de datos individual.

6. De los pacientes con DM, se identificarán a aquellos que presenten datos de PD, para lo que el investigador principal se coordinará con el especialista vascular para realización de diagnóstico de PD mediante exploración física y se calculará una kappa con llenado de información en hoja de datos. Posterior a la valoración de especialista vascular se procederá a toma de muestra única por personal capacitado (enfermería) el cual incluirá perfil lipídico (que incluirá colesterol y triglicéridos séricos) y se enviará toma de muestra con la información individual de cada paciente a laboratorio del hospital. Se recabarán dichos resultados posterior a tiempos ya establecidos de laboratorio hospitalario y se continuara vaciamiento de datos en hojas de recolección.

7. La exploración física y recolección de datos personales será en un horario aleatorio de lunes a viernes en servicio de urgencias, en los horarios de servicio de médico de base especialista vascular avalado para ejercer en HGZ \#50.

8. Se revisara expediente clínico en urgencias en búsqueda de resultado de laboratorio sérico lipídico (triglicéridos y colesterol) con su posterior vaciado numérico en hoja de datos; en caso de estar ausentes, se procederá a toma de muestra por personal capacitado de enfermería o personal médico mediante jeringa de 5 o $10 \mathrm{ml}$ con técnica habitual y llenado de tubo rojo, su rotulación con el nombre de paciente para continuar su procesamiento en área de análisis de laboratorio del HGZ\#50 con el equipo de modelo de serie: CA6000, y su posterior vaciamiento de resultados en hoja de datos.

9. Se realizará vaciado sistemático de resultados obtenidos en la hoja de 
recolección de datos de factores cardiovasculares y presencia de PD.

10. Se compararán los factores cardiovasculares de pacientes con diabetes mellitus sin pie diabético y los que presentan PD.

11. Una vez completada la muestra recomendada se realizará análisis estadístico, elaboración de gráficas y cuadros de los resultados obtenidos.

12. Posteriormente se organizará y realizará presentación e interpretación de datos obtenidos.

13. Se realizará difusión de los resultados a las autoridades del HGZ \#50, así como publicación del trabajo.

\section{CONSIDERACIONES ÉTICAS}

El presente estudio se realizó de acuerdo con lo que dispone el Reglamento de la Ley General de Salud en materia de investigación, Secretaria de Salud (1984), específicamente en los siguientes apartados: en relación con el artículo 14, fracciones V, VII, Y VIII.

Este trabajo se apega a lo establecido en la Ley General de Salud, en su título V, Art 100 , este protocolo de investigación se desarrollará conforme a lo siguiente:

1. Deberá adaptarse a los principios científicos y éticos que justifican la investigación médica, especialmente en lo que se refiere a su posible contribución A la solución de problemas de salud y al desarrollo de nuevos campos de la ciencia médica.

2. Podrá realizarse sólo cuando el conocimiento que se pretenda producir no pueda obtenerse por otro método idóneo.

Podrá efectuarse sólo cuando exista una razonable seguridad de que no expone a riesgos ni a daños innecesarios al sujero en experimentación.

3. Sólo podrá realizarse por profesionales de la salud en instituciones médicas que actúen bajo la vigilancia de las autoridades sanitarias competentes.
4. Las demás que establezca la correspondiente reglamentación.

ARTíCULO 17. Se considera como riesgo de la investigación a la probabilidad de que el sujeto de investigación sufra algún daño como consecuencia inmediata o tardía del estudio.

En este caso, el estudio es de riesgo tipo II: investigación con riesgo mínimo por la toma de muestra de laboratorio de vena periférica. Estudios prospectivos que emplean el riesgo de datos a través de procedimientos comunes en exámenes físicos o psicológicos de diagnósticos 0 tratamiento rutinarios.

El protocolo de investigación será revisado por el comité de investigación y ética médica local para su autorización y validación previa. Se garantiza la confidencialidad de los datos obtenidos en el presente estudio, los cuales serán utilizados exclusivamente para este protocolo.

Acatando el Artículo 21 previo a la explicación del objetivo del estudio, se solicitó el consentimiento informado de los sujetos de investigación y de acuerdo con la fracción I, IV, VI, VII, VIII, se aclararon las dudas que surgieron al momento de la entrevista en cada una de las preguntas del instrumento. La información proporcionada se manejó en forma confidencial.

\section{ANÁLISIS ESTADÍSTICO}

Se incluyeron pacientes adultos en área de Urgencias de HGZ\#50 IMSS SLP con diagnóstico de PD de diciembre 2018 a enero 2019.

Se estudiaron las variables: presencia de PD, IMC, género, edad, tabaquismo, HTA y dislipidemia.

Se utilizó R Studio 1.0.153, se determinó normalidad y se realizaron correlaciones $y$ comparaciones entre las variables.

\section{RESULTADOS}

Se trata de un estudio observacional, Analítico y trasversal de 201 pacientes con diagnóstico de DM realizado en el HGZ \#50 
TABLA 1. 3. Frecuencia de edad, estatura, peso e IMC

\begin{tabular}{|l|c|c|c|c|c|c|c|}
\hline & Mean & Sd. & Min & Median & Max & $\mathrm{n}$ & IQR \\
\hline Edad & 59.4 & 15.3 & 18.0 & 60.0 & 97.0 & 201 & 19.0 \\
\hline Estatura & 1.6 & 0.1 & 1.4 & 1.6 & 2.0 & 201 & 0.1 \\
\hline Peso & 71.3 & 15.1 & 4.0 & 71.0 & 121.0 & 201 & 21.0 \\
\hline IMC & 27.0 & 5.3 & 1.0 & 27.0 & 45.8 & 201 & 7.0 \\
\hline
\end{tabular}

Fuente: Dr. Juan Manuel López Días/ Dr. Juan Pablo García Ugalde

en un rango de edad de 18 a 97 años, con una media de 59.4 y mediana de 60 años, con un rango Intercuantil (IQR) de 19 y desviación standard (DS) de 15.3. Con relación a su IMC (Peso en Kilogramos/ talla en metros al cuadrado) tuvo un rango de 45.8 a $17 \mathrm{~kg} / \mathrm{m} 2$, con una media de 27 $\mathrm{kg} / \mathrm{m} 2$ y mediana de $27.1 \mathrm{~kg} / \mathrm{m} 2$, un rango IQ de 7 y una DS de 5.3 (TABLA 1.3).

Del total de los 201 pacientes de este estudio, el $48.76 \%$ (98 pacientes) fueron de género masculino y $51.24 \% \quad(103$ pacientes) de género femenino (TABLA 1. 4).

TABLA 1.4. Frecuencia de género

\begin{tabular}{|c|c|c|}
\hline & Frecuencia & Porcentaje \\
\hline Hombre & 98 & 48.76 \\
\hline Mujer & 103 & 51.24 \\
\hline Total & 201 & 100.0 \\
\hline \multicolumn{2}{|l|}{$\begin{array}{l}\text { Fuente: Dr. Juan Manuel López Días/ Dr. Juan Pablo } \\
\text { García Ugalde }\end{array}$} \\
\hline
\end{tabular}

De los pacientes con DM de este estudio, la prevalencia total que presentó PD fue de $31.34 \%$ (63 pacientes) y $68.66 \%$ (138 pacientes) que no integraban el diagnostico de PD (TABLA 1.5).

TABLA 1.5. Frecuencia de pie diabético

\begin{tabular}{|c|c|c|}
\hline & Frecuencia & Porcentaje \\
\hline Hombre & 138 & 68.66 \\
\hline Mujer & 63 & 31.34 \\
\hline Total & 201 & 100.1 \\
\hline
\end{tabular}

Fuente: Dr. Juan Manuel López Días/ Dr. Juan Pablo García Ugalde

El total de nuestros pacientes con DM fueron agrupados según el grado de IMC, siendo el grupo de sobrepeso y peso adecuado los mas frecuentes en nuestra población representando un $35.32 \%$ cada uno de los grupos (representando 71 pacientes cada uno) seguido del grupo de obesidad grado I (con IMC 25-30 kg/m2) con un 20.8\% (representado por 42 pacientes), siendo desnutrición y obesidad grado II y III los grupos menos frecuentes con $4.48 \%, \quad 2,98 \%$ y $2,98 \%$ respectivamente (TABLA 1.6).

TABLA 1.6. Frecuencia de la variable índice de masa corporal.

\begin{tabular}{|c|c|c|}
\hline & Frecuencia & Porcentaje \\
\hline Desnutrición & 5 & 2.488 \\
\hline Peso adecuado & 71 & 35.323 \\
\hline Sobrepeso & 71 & 35.323 \\
\hline Obesidad I & 42 & 20.896 \\
\hline Obesidad II & 6 & 2.985 \\
\hline Obesidad III & 6 & 2.985 \\
\hline Total & 201 & 100 \\
\hline
\end{tabular}

Fuente: Dr. Juan Manuel López Días/ Dr. Juan Pablo García Ugalde

Del total de los 201 se identificaron las siguientes categorías: sobrepeso con un $62.19 \%$ (125 pacientes), HTA sistémica un $72.14 \%$ de nuestra población(145 pacientes), tabaquismo en un $44.7 \%$ (90 pacientes) y dislipidemia (DL) en un 53\%(107 pacientes); siendo la HTA sistémica seguida por sobrepeso las categorías presentes en más de la mitad de nuestra población (TABLA 1.7).

Se analizó la relación de la edad de pacientes con y sin PD utilizando la prueba T de Student con una media de 58.5 y DS de 15.6 para pacientes sin PD y una media de edad de 61.3 y una DS de 14.6 para pacientes con $\mathrm{PD}$, teniendo un $\mathrm{P}=0.2172$ 
TABLA 1. 7. Frecuencia de las variables

\begin{tabular}{|c|c|c|}
\hline & Frecuencia & Porcentaje \\
\hline \multicolumn{3}{|c|}{ Sobrepeso } \\
\hline No & 76 & 37.81 \\
\hline $\mathrm{Si}$ & 125 & 62.19 \\
\hline Total & 201 & 100.00 \\
\hline \multicolumn{3}{|c|}{ Tabaquista } \\
\hline No & 111 & 55.22 \\
\hline $\mathrm{Si}$ & 90 & 44.78 \\
\hline Total & 201 & 100.00 \\
\hline \multicolumn{3}{|l|}{ HTA } \\
\hline No & 56 & 27.86 \\
\hline Si & 145 & 72.14 \\
\hline Total & 201 & 100.00 \\
\hline \multicolumn{3}{|c|}{ Dislipidemia } \\
\hline No & 94 & 46.77 \\
\hline $\mathrm{Si}$ & 107 & 53.23 \\
\hline Total & 201 & 100 \\
\hline
\end{tabular}

Fuente: Dr. Juan Manuel López Días/ Dr. Juan Pablo García Ugalde

no significativa (Ver Anexo GRÁFICA 1. 1).

En cuanto a la asociación de la variable sexo en relación con la presencia de PD fue analizada mediante Odds Ratio (OR) en la cual 63 pacientes presentaron PD siendo $52.38 \%$ mujeres y $47.62 \%$ hombres en comparación a pacientes sin PD (total de 138 pacientes) siendo pacientes mujeres de $50.72 \%$ y hombres en un $49.28 \%$ obteniendo resultados con un OR de 1.068197 con un intervalo de confianza (IC) de $95 \%$ siendo un resultado no significativo con $\mathrm{P}=0.8796505$ (Ver Anexo GRÁFICA

1. 2).

Se realizó la asociación de la variable IMC desglosada por categorías (peso normal, sobrepeso, obesidad grado: I,II,III) en relación a los paciente con y sin PD en pacientes, resultando en la población sin PD (138 pacientes en total) el predominio con sobrepeso (54 pacientes para un
$39.13 \%$ ) seguido por peso normal (43 paciente para un $31 \%$ ) y obesidad grado (con 28 pacientes para un $20.29 \%$ ) seguidos por obesidad grado III, desnutrición y obesidad grado II con un $6 \%$, $4 \%$ y $3 \%$ respectivamente.

En lo que corresponde a pacientes con PD (un total de 63 pacientes) en el cual el $44 \%$ (28 pacientes) tenían peso normal o adecuado siendo seguidos por pacientes con sobrepeso y obesidad (con 17 pacientes para $26 \%$ y 14 pacientes para un $22 \%$ ) en su gran mayoría, el resto 3 pacientes con obesidad grado II y 1 paciente con desnutrición (Ver Anexo GRÁFICA 1. 3).

En cuanto a la asociación de pacientes con PD que tenían sobrepeso u obesidad (34 pacientes con PD vs 91 pacientes sin PD) en relación a pacientes sin sobrepeso (29 pacientes con PD vs 47 pacientes sin PD) se analizó a través de la prueba $X^{2}$ de Pearson para independencia de dos variables, donde se utilizó corrección de Yates con resultado de $X^{2}: 2.152635$ para una p: 0.142325 la cual no fue significativa, sin embargo es indicativa de tendencia (Ver Anexo GRÁFICA 1. 4).

Se realizó la valoración de asociación de pacientes con PD con la variable de HTA (55 pacientes con HTA vs 8 pacientes sin HTA, para un $27 \%$ y un $3.9 \%$ respectivamente) en relación a pacientes sin PD (90 pacientes con HTA vs 48 pacientes sin HTA) analizándose mediante prueba $X^{2}$ de Pearson para independencia de dos variables con corrección de Yates para un resultado de $X^{2}: 9.425937$ para una p: 0.002139378 la cual fue significativa por lo que podemos rechazar la hipótesis nula con un nivel de significación del 99\%; posteriormente se realizó la Prueba Exacta de Fisher para significancia estadística con $\mathrm{p}$ : $\quad 0.00115695 \quad$ estadísticamente significativa, para un intervalo de confianza del 95\% (1.556-9.603) y un Odds Ratio estimado de 3.645603 (Ver Anerxo GRÁFICA 1. 5). 
Se valoró la asociación del tabaquismo con la presencia de PD (37 pacientes sin tabaquismo vs 26 pacientes con tabaquismo positivo, para un porcentaje de $12.9 \%$ y $18.4 \%$ respectivamente) en contraste en pacientes sin PD (74 pacientes con tabaquismo negativo para un $36 \%$ y 64 pacientes con tabaquismo positivo representando el 31\%). Realizándose prueba $X^{2}$ de Pearson para independencia de dos variables con valor de 0.4562227 siendo estadísticamente no significativa con una p: 0.4993946. (GRÁFICA 1. 6).

Se analizó la asociación de dislipidemia (DL) en los pacientes con PD (30 pacientes con DL para un $14 \%$ y 33 pacientes sin DL para un porcentaje del 16\%) y sin PD (64 pacientes sin DL y 74 pacientes con DL para un porcentaje de $31 \%$ y $36 \%$ respectivamente). Con realización de prueba $X^{2}$ de Pearson para independencia de dos variables con un valor de 0.02681096 y una p: 0.8699354 estadísticamente no significativa (GRÁFICA 1. 7).

\section{DISCUSIÓN}

En nuestro estudio se observó una prevalencia de hasta $31 \%$ pacientes con PD lo cual se encuentra dentro de lo reportado por Saba Noor y Col, 2015 quienes concuerdan con una prevalencia mayor al $25 \%$ de igual manera reportado en la epidemiologia de la guía de práctica clínica SS-005-08 de prevención, diagnóstico y tratamiento oportuno del PD en el primer nivel de atención 2012 de hasta $25 \%$.

En el presente estudio por lo que respecta a la presencia de las variables de tabaquismo, IMC y el género no tuvieron significancia estadística su asociación con PD; lo cual ya había sido descrito y reportado por Hajieh Shahbazian y Col, en 2013 en su estudio analítico y descriptivo en pacientes con PD con 430 pacientes, de acuerdo al consenso internacional del International Working Group on Diabetic Foot (IWGDF) sin embargo todos sus pacientes tenían historia o cursaban ya con PD.

La DL en contexto de hipertrilgiceridemia o hipercolesterolemia en el presente estudio resulto estadísticamente no significativa ya previamente descrita por Laura N. McEwen y Col. en 2013 en su estudio observacional en pacientes con PD dividiéndolos en 4 fenotipos: presencia de pie de Charcot, PD con debridación, PD con amputación y sin condiciones agregadas.

Es importante señalar la presencia de un resultado estadísticamente significativo que obtuvo una buena asociación con el PD: la HTA sistémica, la cual concuerda en un estudio retrospectivo de cohorte realizado previamente por Khalid Al-Rubeaan y Col. en 2015 en pacientes 62,681 pacientes con PD únicamente (con estatus de: ulcerado, con presencia de gangrena o amputación) el cual se relaciona de manera directa con la EAP por su contexto como uno de los principales desencadenantes primarios para PD. Una de las diferencias en relación con revisiones, estudios observacionales y retrospectivos fue la comparación de pacientes sin presencia de PD en contra de pacientes con diagnóstico actual o historia de PD para buscar su asociación como probable factor desencadenante, lo cual nos coloca en un punto de vista diferente a la literatura existente, la cual engloba los factores de riesgo asociado a complicaciones agregadas a nuestra patología de estudio ya existente.

Limitaciones y/o nuevas perspectivas de investigación

Limitaciones: se eliminaron del estudio los pacientes en los cuales no se tenían completas todas las variables necesarias reduciendo el número total de pacientes ingresados al estudio.

Nuevas Perspectivas: el presente estudio sirve como base para nuevos protocolos donde se estudie la relación directa entre PD e sistémica tanto para su abordaje en primer nivel hasta su asociación con la morbimortalidad, los cuales pueden ser prospectivos de mayor duración. Durante la realización del estudio se hizo diagnóstico de Novo en pacientes que no se conocían con PD por lo que se estima que la 
patología continúa siendo subdiagnosticada desde unidades de primer nivel.

\section{CONCLUSIONES}

En nuestro estudio no se demostró una asociación estadísticamente significativa sobre el pie diabético de las variables señaladas en la literatura universal como factores de riesgo cardiovascular: tabaquismo, sobrepeso-obesidad 0 dislipidemia, sin embargo, la hipertensión arterial sistémica sí tuvo correlación estadísticamente significativa con la presencia de pie diabético.

Se necesitan más estudios para determinar apropiadamente la causalidad de la hipertensión sobre el pie diabético, sin embargo, es una pieza importante para el abordaje y manejo guiado de forma multidisciplinaria.

\section{REFERENCIAS}

1. William TC GB. Standards of Medical Care in Diabetes. Diabetes Care. 2017 Enero; 40(1). Disponible en: http://www.epi.uff.br/wp-content/uploads/2013/10/dc 40 s1 final.pdf

2. Nagase, Takashi et al. Screening of Foot Inflammation in Diabetic Patients by Non-Invasive Imaging Modalities. Global Perspective on Diabetic Foot Ulcerations. 2012. Disponible en: https://www.semanticscholar.org/paper/3-Screening-of-Foot-Inflammation-in-Diabetic-by-NagaseSanada/8a9d54c32f368b53a329e2bb6349abe7ca6cab11

3. Cheng AY. Introduction. Canadian Journal of Diabetes. 2013 04;37:S1-S3. https://doi.org/10.1016/j.jcjd.2013.01.009

4. Rangel JT. Proporción de incidencia de amputaciones en pacientes con lesiones del pie diabético. Revista Mexicana de Angiologia. 2015 Enero; 43(1). Disponible en: http://www.medigraphic.com/cgi$\underline{\mathrm{bin} / \mathrm{new} / \text { resumen.cgi?IDARTICULO }=57567}$

5. Namgoong S, Jung S, Han S, Jeong S, Dhong E, Kim W. Risk factors for major amputation in hospitalised diabetic foot patients. International Wound Journal. 2015 Oct 19;13:13-19. https://doi.org/10.1111/iwj.12526

6. Bus SA, van Netten JJ, Lavery LA, Monteiro-Soares M, Rasmussen A, Jubiz Y, Price PE, . IWGDF guidance on the prevention of foot ulcers in at-risk patients with diabetes. Diabetes/Metabolism Research and Reviews. 2016 01;32:16-24. https://doi.org/10.1002/dmrr.2696

7. Tratamiento de la Diabetes Mellitus tipo 2 en el primer nivel de el primer nivel de Atención. Instituto Mexicano del Seguro Social. 2014.2 Disponible en: http://www.cenetec.salud.gob.mx/descargas/gpc/CatalogoMaestro/718 GPC Tratamiento de diabet es mellitus tipo $2 / 718 \mathrm{GER} . \mathrm{pdf}$

8. Pinto A, Tuttolomondo A, Di Raimondo D, Fernandez P, La Placa S, Di Gati M, Licata G. Cardiovascular risk profile and morbidity in subjects affected by type 2 diabetes mellitus with and $\begin{array}{llll}\text { without diabetic } & & \\ \end{array}$ https://doi.org/10.1016/j.metabol.2008.01.004

9. Tuttolomondo A, Maida C, Pinto A. Diabetic Foot Syndrome as a Possible Cardiovascular Marker in Diabetic Patients. Journal of Diabetes Research. 2015;2015:1-12. https://doi.org/10.1155/2015/268390

10. Hung S, Huang Y, Hsu L, Chen C, Yang H, Sun J, Lin C, Wang C. Treatment for Diabetic Foot Ulcers Complicated by Major Cardiac Events. Canadian Journal of Diabetes. 2015 06;39(3):183-187. https://doi.org/10.1016/j.jcjd.2014.11.002 
11. Dietrich I, Braga GA, de Melo FG, da Costa Silva Silva ACC. The Diabetic Foot as a Proxy for Cardiovascular Events and Mortality Review. Current Atherosclerosis Reports. 2017 Oct 02;19(11). https://doi.org/10.1007/s11883-017-0680-z

12. Husten CG. How should we define light or intermittent smoking? Does it matter?. Nicotine \& Tobacco Research. 200903 05;11(2):111-121. https://doi.org/10.1093/ntr/ntp010

13. U.S. Department of Health and Human. The Health consequences of smoking-50 years of progress. A report of the Surgeon General. Centers for Disease Control and Prevention and Health Promotion. 2014. Disponible en: https://www.surgeongeneral.gov/library/reports/50-years-of-progress/index.html

14. Morris PB, Ference BA, Jahangir E, Feldman DN, Ryan JJ, Bahrami H, El-Chami MF, Bhakta S, Winchester DE, Al-Mallah MH, Sanchez Shields M, Deedwania P, Mehta LS, Phan BAP, Benowitz NL. Cardiovascular Effects of Exposure to Cigarette Smoke and Electronic Cigarettes. Journal of the $\begin{array}{llll}\text { American } \quad \text { College } & 2015 \quad \text { of } & \text { Cardiology. } 12): 1378-1391 .\end{array}$ https://doi.org/10.1016/j.jacc.2015.07.037

15. Clayton W, Elasy T. A review of the Pathophysiology, classification, and treatment of foot ulcers in Diabetic Patients. Clinical Diabetes. 2009; 27(2). Disponible en: https://www.semanticscholar.org/paper/A-review-of-the-Pathophysiology-\%2C-classification-\%2CClayton-Elasy/cc757ade5358ee67d2f61fa8816feaf9c0ebedb1

16. Rebolledo FA. The Pathogenesis of the Diabetic Foot Ulcer: Prevention and Management. Global Perspective on Diabetic Foot Ulcerations. 2011; 9(29). Disponible en: https://pdfs.semanticscholar.org/50ce/4b622a87ebfc4a75ddbc1e2ff490b98c6acf.pdf? ga=2.5832906 9.616562820.1551309255-1581848156.1551028403

17. Martínez F, Guerrero G, Ochoa P, Anaya R, Muñoz J. Diagnóstico, clasificación y tratamiento de las infecciones en pie diabético. Cirugia General. 2012; 34(32). Disponible en: http://www.medigraphic.com/cgi-bin/new/resumen.cgi?IDARTICULO=39853

18. Brownrigg J, Apelqvist J, Bakker K, Schaper N, Hinchliffe R. Evidence-based Management of PAD \& the Diabetic Foot. European Journal of Vascular and Endovascular Surgery. 2013 06;45(6):673-681. https://doi.org/10.1016/j.ejvs.2013.02.014

19. Sinwar PD. The diabetic foot management - Recent advance. International Journal of Surgery. 2015 03;15:27-30. https://doi.org/10.1016/j.ijsu.2015.01.023

20. Kumar A. A New Classification of Diabetic Foot Complications: A simple and effective teaching tool. Journal of Diabetic Foot Complications. 2012; 4(1). Disponible en: http://jdfc.org/2012/volume-4-issue1/a-new-classification-of-diabetic-foot-complications-a-simple-and-effective- 20. Kumar A. A New Classification of Diabetic Foot Complications: A simple and effective teaching tool. Journal of Diabetic Foot Complications. 2012; 4(1). Disponible en: http://jdfc.org/2012/volume-4-issue-1/a-newclassification-of-diabetic-foot-complications-a-simple-and-effective-teaching-tool/

21. Bakker K, Apelqvist J, Schaper NC, . Practical guidelines on the management and prevention of the diabetic foot 2011. Diabetes/Metabolism Research and Reviews. 201201 23;28:225-231. https://doi.org/10.1002/dmrr.2253

22. Van Netten JJ, Price PE, Lavery LA, Monteiro-Soares M, Rasmussen A, Jubiz Y, Bus SA, . Prevention of foot ulcers in the at-risk patient with diabetes: a systematic review. Diabetes/Metabolism Research and Reviews. 2016 01;32:84-98. https://doi.org/10.1002/dmrr.2701

23. Jiang HJ, Stryer D, Friedman B, Andrews R. Multiple Hospitalizations for Patients With Diabetes. Diabetes Care. 200305 01;26(5):1421-1426. https://doi.org/10.2337/diacare.26.5.1421 
24. Prevención, diagnóstico y tratamiento oportuno del pie diabético en el primer nivel de atención. Secretaria de Salud. 2012. Disponible en: https://cenetec-difusion.com/gpc-sns/?p=660

25. Sohn M, Meadows JL, Oh EH, Budiman-Mak E, Lee TA, Stone NJ, Pearce WB. Statin use and lower extremity amputation risk in nonelderly diabetic patients. Journal of Vascular Surgery. 2013 Dec;58(6):1578-1585.e1. https://doi.org/10.1016/j.jvs.2013.06.069

26. Bus SA, van Netten JJ. A shift in priority in diabetic foot care and research: $75 \%$ of foot ulcers are preventable. Diabetes/Metabolism Research and Reviews. 2016 01;32:195-200. https://doi.org/10.1002/dmrr.2738

27. Brownrigg J, Apelqvist J, Bakker K, Schaper N, Hinchliffe R. Evidence-based Management of PAD \& the Diabetic Foot. European Journal of Vascular and Endovascular Surgery. 2013 06;45(6):673-681. https://doi.org/10.1016/i.ejvs.2013.02.014

28. Tuttolomondo A, Maida C, Pinto A. Diabetic Foot Syndrome as a Possible Cardiovascular Marker in Diabetic Patients. Journal of Diabetes Research. 2015;2015:1-12. https://doi.org/10.1155/2015/268390

29. William T. Cefalu GB. Standards of Medical Care in Diabetes. Diabetes care. 2017; 40(1). Disponible

en: http://care.diabetesjournals.org/content/diacare/suppl/2016/12/15/40.Supplement 1.DC1/DC 40 S1 final.pdf 


\section{ANEXO}

GRAFICO 1. Análisis de Asociación por Edad

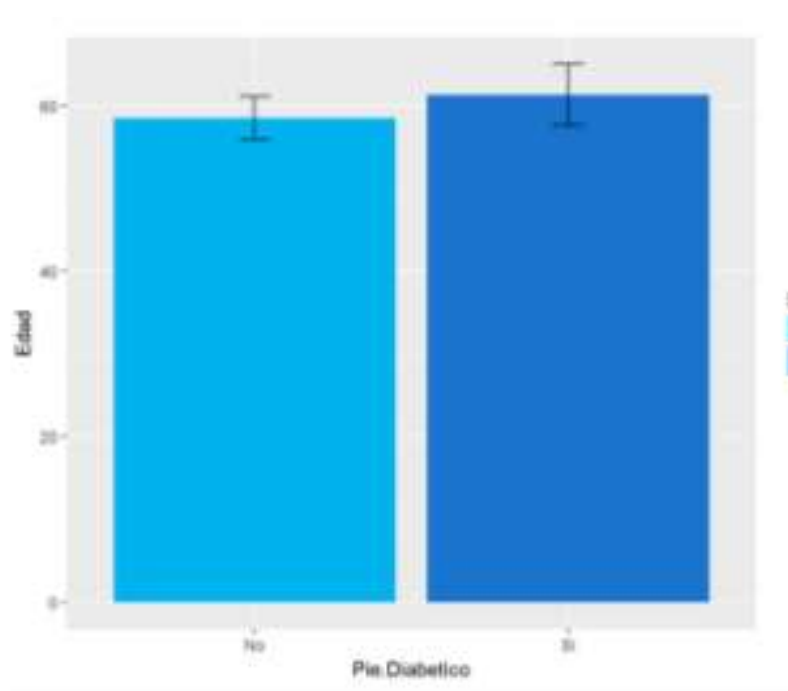

\begin{tabular}{|l|c|c|c|c|c|c|c|}
\hline \multicolumn{10}{|c|}{ No Pie diabético } \\
\hline & mean & sd & min & median & max & $\mathrm{n}$ & IQR \\
\hline Edad & 58.5 & 15.6 & 18.0 & 59.0 & 97.0 & 138 & 19.0 \\
\hline $\begin{array}{l}\text { Fuente: Dr. Juan Manuel López Días/ Dr. Juan Pablo García } \\
\text { Ugalde }\end{array}$ \\
\hline
\end{tabular}

\begin{tabular}{|c|c|c|c|c|c|c|c|}
\hline \multicolumn{10}{|c|}{ Pie diabético } \\
\hline & mean & sd & min & median & max & $\mathrm{n}$ & IQR \\
\hline Edad & 61.3 & 14.6 & 23.0 & 62.0 & 84.0 & 63 & 21.0 \\
\hline
\end{tabular}

Fuente: Dr. Juan Manuel López Días/ Dr. Juan Pablo García Ugalde

GRAFICO 2. Análisis de Asociación por Sexo



No

\begin{tabular}{|c|c|c|}
\hline No Pie diabético & Frecuencia & Porcentaje \\
\hline Hombre & 68 & 49.28 \\
\hline Mujer & 70 & 50.72 \\
\hline Total & 138 & 100.00 \\
\hline \multicolumn{2}{|c|}{$\begin{array}{l}\text { Fuente: Dr. Juan Manuel López Días/ Dr. Juan Pablo } \\
\text { García Ugalde }\end{array}$} \\
\hline
\end{tabular}

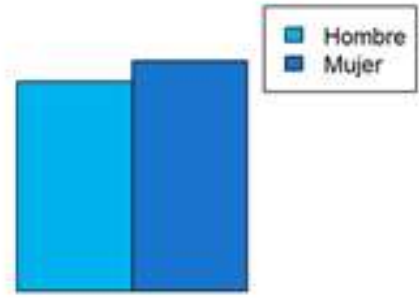

Pie Diabetico

Fuente: Dr. Juan Manuel López Días/ Dr. Juan Pablo García Ugalde

\begin{tabular}{|c|c|c|}
\hline Pie diabético & Frecuencia & Porcentaje \\
\hline Hombre & 68 & 49.28 \\
\hline Mujer & 70 & 50.72 \\
\hline Total & 138 & 100.00 \\
\hline $\begin{array}{l}\text { Fuente: Dr. Juan Manuel López Días/ Dr. Juan Pablo } \\
\text { García Ugalde }\end{array}$ \\
\hline
\end{tabular}


GRAFICO 3. Indice de masa corporal por categorias

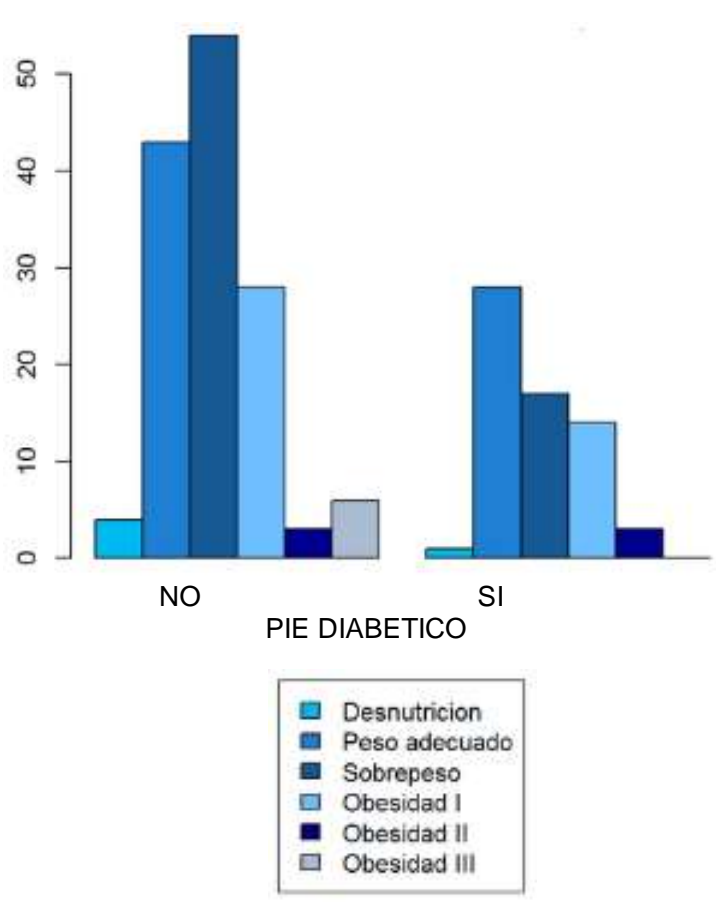

\begin{tabular}{|c|c|c|}
\hline No Pie diabético & Frecuencia & Porcentaje \\
\hline Desnutrición & 4 & 2.899 \\
\hline Peso adecuado & 43 & 31.159 \\
\hline Sobrepeso & 54 & 39.130 \\
\hline Obesidad I & 28 & 20.290 \\
\hline Obesidad II & 3 & 2.174 \\
\hline Obesidad III & 6 & 4.348 \\
\hline Total & 138 & 100.000 \\
\hline
\end{tabular}

\begin{tabular}{|c|c|c|}
\hline Pie diabético & Frecuencia & Porcentaje \\
\hline Desnutrición & 1 & 1.587 \\
\hline Peso adecuado & 28 & 44.444 \\
\hline Sobrepeso & 17 & 26.984 \\
\hline Obesidad I & 14 & 22.222 \\
\hline Obesidad II & 3 & 4.762 \\
\hline Obesidad III & 0 & 0.000 \\
\hline Total & 63 & 100.000 \\
\hline
\end{tabular}

Fuente: Dr. Juan Manuel López Días/ Dr. Juan Pablo García Ugalde

GRAFICO 4. Pie diabético por sobrepeso

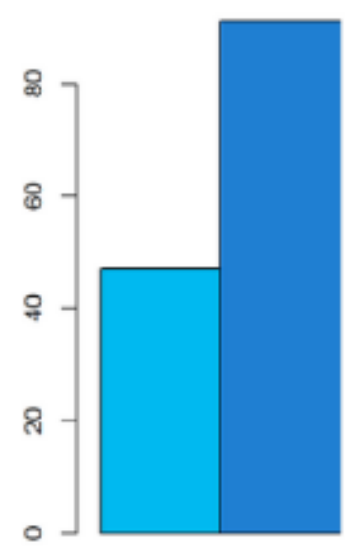

NO

PIE DIABETICO

\begin{tabular}{|c|c|c|c|}
\hline \multicolumn{4}{|c|}{ No Pie diabético } \\
\hline Data: sobrepeso & NO & SI & Row total \\
\hline Count & 47 & 29 & \multirow{7}{*}{$\begin{array}{c}76 \\
37.811 \%\end{array}$} \\
\hline Expected values & 52.179 & 23.821 & \\
\hline $\begin{array}{c}\text { Chi-square } \\
\text { constribution }\end{array}$ & 0.514 & 1.126 & \\
\hline Row percent & $61.842 \%$ & $38.158 \%$ & \\
\hline Column percent & $34.058 \%$ & $46.032 \%$ & \\
\hline Total percent & $23.383 \%$ & $14.428 \%$ & \\
\hline Std residual & 0.717 & 1.061 & \\
\hline
\end{tabular}

Fuente: Dr. Juan Manuel López Días/ Dr. Juan Pablo García Ugalde

\begin{tabular}{|c|c|c|c|}
\hline \multicolumn{3}{|c|}{ Pie diabético } \\
\cline { 1 - 3 } Data sobrepeso & NO & SI & Row total \\
\hline Count & 91 & 34 & \\
\cline { 1 - 3 } Expected values & 85.821 & 39.179 & \\
\cline { 1 - 3 } Chi-square constribution & 0.313 & 0.685 & \multirow{2}{*}{125} \\
\cline { 1 - 3 } Row percent & $72.800 \%$ & $27.200 \%$ & \multirow{2}{*}{$62.189 \%$} \\
\cline { 1 - 3 } Column percent & $65.942 \%$ & $53.968 \%$ & \\
\cline { 1 - 3 } Total percent & $45.274 \%$ & $16.915 \%$ & \\
\hline Std residual & 0.559 & 0.827 & \\
\cline { 1 - 3 } Column total & 138 & 63 & \multirow{2}{*}{201} \\
& $68.657 \%$ & $31.343 \%$ & \\
\hline
\end{tabular}

Fuente: Dr. Juan Manuel López Días/ Dr. Juan Pablo García Ugalde

Fuente: Dr. Juan Manuel López Días/ Dr. Juan Pablo García Ugalde 
GRAFICO 5. Pie diabético por HTA



NO

PIE DIABETICO

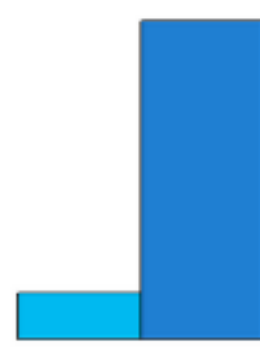

SI

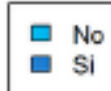

No Pie diabético

\begin{tabular}{|c|c|c|c|}
\hline Data: HTA & NO & SI & Row total \\
\cline { 1 - 3 } Count & 48 & 8 & \\
\cline { 1 - 3 } Expected values & 38.448 & 17.552 & \\
\cline { 1 - 3 } Chi-square constribution & 2.373 & 5.198 & \multirow{2}{*}{56} \\
\cline { 1 - 3 } $27.861 \%$ \\
\cline { 1 - 3 } Row percent & $85.715 \%$ & $14.286 \%$ & \\
\cline { 1 - 3 } Column percent & $34.783 \%$ & $12.698 \%$ & \\
\cline { 1 - 3 } Total percent & $23.881 \%$ & $3.980 \%$ & \\
\cline { 1 - 3 } Std residual & 1.541 & -2.280 & \\
\cline { 1 - 3 } & &
\end{tabular}

Fuente: Dr. Juan Manuel López Días/ Dr. Juan Pablo García Ugalde

\begin{tabular}{|c|c|c|c|}
\hline \multicolumn{3}{|c|}{ Pie diabético } \\
\cline { 1 - 3 } Data: HTA & NO & SI & Row total \\
\hline Count & 90 & 55 & \\
\cline { 1 - 3 } Expected values & 99.552 & 45.448 & \\
\cline { 1 - 3 } Chi-square constribution & 0.917 & 2.008 & \multirow{2}{*}{145} \\
\cline { 1 - 3 } Row percent & $62.069 \%$ & 37.931 & \\
\cline { 1 - 3 } Column percent & $65.217 \%$ & 87.302 & \\
\hline Total percent & $44.776 \%$ & 27.363 & \\
\cline { 1 - 3 } Std residual & -0.957 & 1.417 & \\
\cline { 1 - 3 } Column total & 138 & 63 & \\
& $68.657 \%$ & 31.343 & \\
\cline { 1 - 3 } & &
\end{tabular}

Fuente: Dr. Juan Manuel López Días/ Dr. Juan Pablo García Ugalde

Fuente: Dr. Juan Manuel López Días/ Dr. Juan Pablo García Ugalde

GRAFICO 6. Pie diabético por tabaquismo

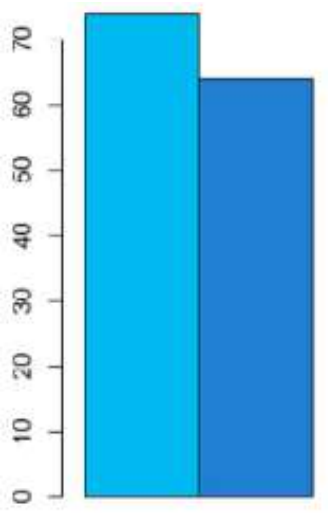

$\mathrm{NO}$

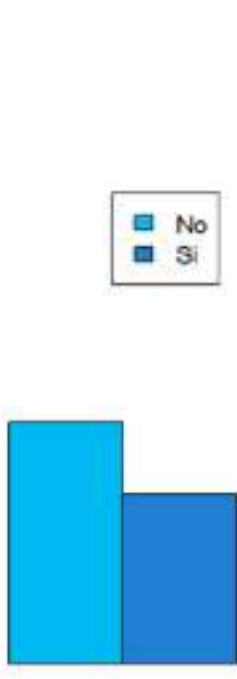

SI

PIE DIABETICO

\begin{tabular}{|l|c|c|c|}
\hline \multicolumn{4}{|c|}{ No Pie diabético } \\
\cline { 1 - 3 } \multicolumn{1}{|c|}{ Data: tabaquismo } & NO & SI & Row total \\
\hline Count & 74 & 37 & \\
\cline { 1 - 3 } Expected values & 76.209 & 34.791 & \\
\cline { 1 - 3 } Chi-square constribution & 0.064 & 0.140 & \multirow{2}{*}{$55.224 \%$} \\
\cline { 1 - 3 } Row percent & $66.667 \%$ & $33.333 \%$ & \\
\cline { 1 - 2 } Column percent & $53.623 \%$ & $58.730 \%$ & \\
\cline { 1 - 3 } Total percent & $36.816 \%$ & $18.408 \%$ & \\
\cline { 1 - 2 } Std residual & -0.253 & 0.375 & \\
\cline { 1 - 3 } & & &
\end{tabular}

Fuente: Dr. Juan Manuel López Días/ Dr. Juan Pablo García Ugalde

\begin{tabular}{|c|c|c|c|}
\hline \multicolumn{4}{|c|}{ Pie diabético } \\
\hline Data: tabaquismo & NO & $\mathrm{SI}$ & Row total \\
\hline Count & 64 & 26 & \multirow{7}{*}{$\begin{array}{c}90 \\
44.776 \%\end{array}$} \\
\hline Expected values & 61.791 & 28.209 & \\
\hline Chi-square constribution & 0.079 & 0.173 & \\
\hline Row percent & $71.111 \%$ & $28.889 \%$ & \\
\hline Column percent & $46.377 \%$ & $41.270 \%$ & \\
\hline Total percent & $31.841 \%$ & $12.935 \%$ & \\
\hline Std residual & 0.281 & -0.416 & \\
\hline Column total & $\begin{array}{c}138 \\
68.657 \%\end{array}$ & $\begin{array}{c}63 \\
31.343 \%\end{array}$ & 201 \\
\hline
\end{tabular}

Fuente: Dr. Juan Manuel López Días/ Dr. Juan Pablo García Ugalde 
GRAFICO 7. Pie diabético

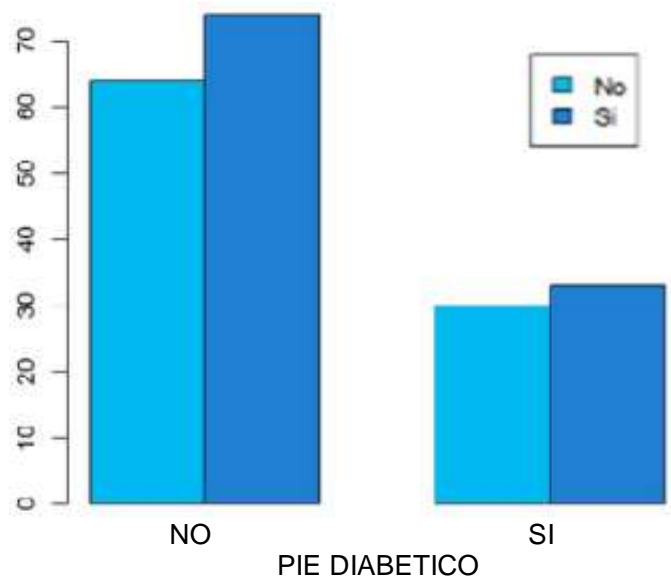

\begin{tabular}{|c|c|c|c|}
\hline \multicolumn{4}{|c|}{ No Pie diabético } \\
\hline Data: dislipidemia & NO & SI & Row total \\
\hline Count & 64 & 30 & \multirow{7}{*}{$\begin{array}{c}94 \\
16.766 \%\end{array}$} \\
\hline Expected values & 64.537 & 29.463 & \\
\hline Chi-square constribution & 0.004 & 0.010 & \\
\hline Row percent & 68.085 & $31.915 \%$ & \\
\hline Column percent & 46.377 & $47.619 \%$ & \\
\hline Total percent & 31.841 & $14.925 \%$ & \\
\hline Std residual & -0.067 & 0.099 & \\
\hline \multicolumn{4}{|c|}{ Fuente: Dr. Juan Manuel López Días/ Dr. Juan Pablo García Ugalde } \\
\hline \multicolumn{4}{|c|}{ No Pie diabético } \\
\hline Data: dislipidemia & NO & $\mathrm{SI}$ & Row total \\
\hline Count & 74 & 33 & \multirow{7}{*}{$\begin{array}{c}107 \\
53.234 \%\end{array}$} \\
\hline Expected values & 73.463 & 33.537 & \\
\hline Chi-square constribution & 0.004 & 0.009 & \\
\hline Row percent & $69.159 \%$ & $30.841 \%$ & \\
\hline Column percent & $53.623 \%$ & $52.381 \%$ & \\
\hline Total percent & $36.816 \%$ & $16.418 \%$ & \\
\hline Std residual & 0.063 & -0.093 & \\
\hline Column total & $\begin{array}{c}138 \\
68.657 \%\end{array}$ & $\begin{array}{c}63 \\
31.343 \%\end{array}$ & 201 \\
\hline
\end{tabular}

Fuente: Dr. Juan Manuel López Días/ Dr. Juan Pablo García Ugalde 